\title{
Introduction
}

This book explores international experiences of institutional reform for sustainable development through a series of case studies, seeking to identify positive principles from existing practice to inform further institutional change. Underlying this investigation is the proposition that countries should be making purposeful efforts to reform environmental and resource management policies and practice, and those in other sectors, consistent with the notion of sustainable development and with commitments made under international agreements at, and subsequent to, the 1992 United Nations Conference on Environment and Development (UNCED).

The institutionalization of the sustainability idea, and its eventual integration as a fundamental and mainstream principle of governance, is a long-term project only recently begun. Arguably sustainability has an inexorable logic, on a plane with other deep social logics such as democracy, justice and human rights. Inevitably, it seems, these central animating ideas of modern societies are all intertwining and inseparable; the identification of these strands may be viewed as part of the definition of a moral rationality for global civilization. However, sustainability has yet to attain the recognition and status of its natural partners at national or global levels. This will require both broad normative change and purposive institutional change, and these are key themes of this book.

Now is certainly an important historical point for humanity with respect to institutional development, and one that demands that we attend closely to the task of better understanding the substance and the ways of our institutions. Sustainability, as a newly recognized strand of the moral fibre by which we propose to survive and prosper in a full world, requires urgent practical development within our institutional systems. How can we purposively develop social rules and organizational structures and relationships to support and promote sustainable behaviour? This is certainly a learning project, and one that must attempt to extract lessons as efficiently as possible both from general institutional experience and from the initial attempts at purposive change. This book is an effort to share the experience of one study that has approached this problem.

The study originated from the perspective of informing institutional and policy change in Australia, utilizing international experiences. As we proceeded, however, it became obvious that the themes and principles 
underlying the issue of institutional change for sustainability are more often universal, even though their translation into a specific context must be closely considered. So, while the Australian setting is used from time to time as a reference point, the analysis, content and conclusions of the book are more global than local. This is to be expected, as sustainability is nothing if not an intrinsically global idea.

\section{THE PLAN OF THE BOOK}

The book is organized into three parts. The first part attempts to provide some mental furniture - a landscape of concepts and language to assist later empirical analysis. This is followed, in Part II, by a set of five case studies (Chapters 3 to 7), and Part III works to distil general lessons from the project.

Chapter 1 attempts first to define some reference points within institutional language and link these conceptually to everyday experience of complex organized society. It explores the notion of institutions and the environment of institutional systems. Second, this chapter investigates notions of policyrelevant learning in an institutional context and how they might relate to the task of institutional learning and development for sustainability policy. Chapter 2 develops a framework for the choice of case studies in institutional change through exploring the practical issues of what it is we might wish to learn about and from where the lessons might come. It explores the meaning of 'sustainable development' and how policy and institutional systems might, and do, respond to its imperatives, using Australian institutional responses as examples. The chapter then develops a set of criteria for the selection of case studies and develops a matrix of analytical targets for the study. Finally the chapter briefly describes the case study topics chosen, along with some that might have been but were not, and how the criteria were applied in the choices made.

The first case study in Part II examines the development of the environmental policies of the European Union (EU) into a major institutional commitment to sustainability principles. The EU represents the most advanced, complex and sustained example of integrated policy and institutional development in the history of modern government. The evolution of EU environmental policy from the time of the Stockholm Conference in 1972 to the Maastricht Treaty of 1992 reflects an official concern for accumulating evidence of environmental damage and normative change particularly in Northern Europe. This same constituency is the source of the 'sustainable development' conceptual synthesis, and since 1992 the EU policy discourse has focused increasingly on sustainable development. The case study traces this developmental path through its core modality, 
the periodic Environmental Action Plan. Complexity of political relationships and governmental structures has led to a variety of modes and paths being explored for the integration of sustainability principles into sectoral policies, and a great richness of experience is only partially uncovered here.

The second case study (Chapter 4) looks at the New Zealand legislative reforms resulting in the Resource Management Act 1991. The Resource Management Act (RMA) began as an attempt to consolidate a large fragmented body of legislation and other institutional arrangements for natural resource management. This reform was carried out concurrently with a structural reform of local government. Twenty major statutes and 50 other laws affecting the environment were replaced by the RMA, and more than six hundred units of local government were reduced to about eighty-six. Resource management and the maintenance of environmental quality were placed at the centre of concerns for local government. Planning and regulation of all land use is subject to the singular purpose of the RMA - the sustainable management of natural and physical resources. The focus of planning and decision making was switched from the activities carried out on land to the effects of land and resource use on the environment. This dual reform structure and policy mandate - held much promise, but this has only been partially realized. Close attention reveals a lapse in policy scrutiny at the centre following the decentralizing reform, and the critical need for ongoing political commitment to the values behind sustainability policy.

The third case study surveys the widespread use of a sustainability-specific institutional innovation. From WCED to UNCED and since, great emphasis has been placed on national scale coordination and integration of policy for sustainable development, and the cooperation of government and civil society in communication and policy development. In response over seventy nations - developed, developing and transitional alike - have established a National Council for Sustainable Development (NCSD) or equivalent body. Coordination is provided through a network serviced by the Earth Council, an NGO. In the ideal form, NCSDs have the following features:

- membership representing different levels of government, research, the private sector and community organizations, and through that membership a network of formal and informal linkages

- a focus on a long term, integrated sustainability agenda, with the ability to maintain a purposeful dialogue and environment of policy learning over time

- sufficient status within the institutional system, including through mandated roles viewed as necessary by others, to have an impact on policy and institutional change. 
Together these functions are necessary and logical expressions of the policy task of sustainability. The core rationale of the NCSD is maintenance of an inclusive policy discourse around the broader field of sustainable development, complementing but not replacing inclusive approaches to subsidiary issues. This study examines the roles adopted by, variability among, and comparative success of examples of NCSDs around the world, assessing strengths and weakness and contribution to advancing the sustainability policy agenda in different national settings.

The fourth case study examines the under-recognised policy integration tool termed Strategic Environmental Assessment (SEA). For three decades environmental impact assessment (EIA) of individual development projects has been central to environmental policy. While important, project-EIA does not attend impacts that are cumulative in space or time or the policy settings that establish the directions and parameters of development. Over the same three decades, and well before the emergence of the sustainable development policy agenda, the need for assessment of policies, plans and programmes (PPPs) above the discrete project level has been recognized and advocated. In its various guises, such Strategic Environmental Assessment proposes to insert environmental considerations into policy development in other sectors.

Project-EIA addresses the more obvious, direct causes of environmental degradation. SEA targets less obvious but crucial indirect causes of unsustainable patterns of production and consumption across all policy sectors, focusing on government policy and legislative proposals (for example, in areas such as tax, trade, transport, regional development). SEA thus represents a prime option for implementing policy integration for sustainability. SEA is recommended in major international agreements, and statutory or policy provisions exist in a number of countries. Experience with sustainable development in the 1990s has strengthened interest in SEA, but there is a clear gap between recommending SEA and actual implementation.

This case study examines SEA as a mechanism for policy integration for sustainability, in theory and in (limited) practice in parts of the world. It offers a summary history of SEA, describes the basic elements of SEA, reviews its status in selected countries and regions, and identifies apparent barriers to implementation. The level of detail is kept to the minimum required for the purpose: more detailed sources are available and cited.

The last of the case studies explores the issue of property rights based policy reform in the sustainability arena. Over the past two decades, coincident with the rise of the sustainability discourse, the application of property rights instruments (PRIs) to natural resource management has been advocated as a means to efficiently allocate scarce resources. PRIs have been implemented in a range of applications including the control of sulphur emissions from fossil fuel burning power stations, in controlling discharges into rivers affecting 
water quality, for the allocation of water abstraction, and most notably in marine fisheries management. They have also been proposed in other areas, including carbon emissions and sequestration, and biodiversity conservation. Although often characterized as 'just another tool in the policy toolbox' the case study argues that PRIs involve a fundamental change in social parameters.

Property rights are primary aspects of institutional systems around which resource use regimes are built. Resulting incentives and behaviour patterns structure social relations and cultural values over the long term. The distributional consequences of access and allocation rights and rules contribute a particular logic to social constructions of fairness and equity. Therefore changes to existing property rights regimes need to be considered carefully in light of the specific social and cultural context.

This case study first presents a framework for understanding the nature of common pool resources and the various property regime types that can apply to their management and use, including private property. PRIs are located within this framework, not as a means of privatizing the commons but as an option for regulation of access and withdrawal rights as operational-level sticks in a bundle of rights associated with common pools. The study next elucidates the origin of PRIs in applied economics and the development of the ideas for application in actual policy making using the 'environmental bottom line' approach.

The chapter then turns to the notion of equity in resource use and its linkages with prevailing property regimes. As party to the sustainability troika of concerns, equity is perhaps the least understood and least considered in policy proposals. We explore the implications of the required integration of equity into policy for change in management regimes brought on by overexploitation of resources. The remainder of the chapter sets out to explore differences in the culture of resource use from frontier to commons and aspects of process and path dependence relevant to the application of PRIs, and considerations of the inherent adaptability of different cultures. These elements serve to construct a broad understanding of the nature of, and potential for, the use of PRIs in sustainability policy.

Part III of the book provides a synthesis from the analysis of the cases. On the basis of the conceptual framework established in Part I, and utilizing lessons from the case studies presented in Part II, this final section derives some principles and distils some positive themes of institutional and policy change for sustainable development from the experiences surveyed. These emergent principles and elements are grouped under the two classes of 'objects of learning' identified in Chapter 1 as targets of this investigation: problem reframing and organizing government. These empirically derived themes are key to understanding both the potential of institutional change 
consistent with the sustainability idea and the nature of reforms reviewed in this research. They are not forms or models of institutional change in themselves but rather conceptual and practical principles crucial to progress in institutional change for sustainability.

Grouped under problem reframing are four elements operating on the formation of an explicit conception of the sustainability problem: the institutional accommodation of a sustainability discourse; normative change; legal change; and international law and policy as drivers. Under organizing government, three characteristics emerged as critical to the organizational logic of sustainability: integration of policy and practice; subsidiarity; and reiteration. The central discussion of this concluding chapter is based around this framework of emergent themes and principles, drawing on the case study material to illustrate and develop the lessons. Following that a brief translation of some of these generic principles into a specific jurisdictional context (Australia) is undertaken to illustrate the practical potential for their application to institutional change and policy learning for sustainable development. 